\title{
Human Papillomavirus (HPV) Vaccination Initiation and Completion Among Adult Males in the United States
}

\author{
Yuqi Guo, PhD and Jessamyn Bowling, PhD
}

Background: Men accounted for $43 \%$ of human papillomavirus (HPV)-related cancer cases, but the HPV vaccination rate of adult males remain low. The purposes of this study were to 1) estimate the initiation and completion rate of HPV vaccine, and 2) analyze factors associated with HPV vaccine initiation and completion among adult males.

Metbod: This study analyzed predisposing factors, enabling factors, reinforcing factors, and situational factors associated with the initiation of HPV vaccine among 3228 male adult participants of the 2018 Behavioral Risk Factor Surveillance System. Two binary logistic regressions were estimated based on the Systems Model of Clinical Preventive Care.

Results: The initiation rate and completion rate of HPV vaccine among males aged 18 to 33 years old was $14.1 \%(n=454)$ and $35.4 \%(n=161)$, respectively. Among male adults in the United States, the likelihood of initiating HPV vaccine significantly associated with age (odds ratio $[0 R]=0.87$, $P<.00)$, having routine checkups $(0 R=1.29, P<.05)$, and having high-HPV-risk behaviors $(0 R=1.53, P<.01)$. However, the likelihood of completing HPV vaccines significantly associated with age $(0 \mathrm{R}=0.85, P<.00)$ and having high-HPV-risk behaviors $(\mathrm{OR}=1.79, P<.01)$.

Conclusions: Having primary health care providers and regular checkups are important to male adults to have HPV vaccines. Promoting access to health care resources may improve current vaccination rates among males. (J Am Board Fam Med 2020;33:592-599.)

Keywords: Access to Health Care, Behavioral Risk Factor Surveillance System, Causality, Logistic Models, Men's Health, Papillomavirus Infections, Papillomavirus Vaccines, Risk Taking

\section{Introduction}

Human papillomavirus (HPV) can cause cervical cancer, as well as some cancers of the oropharynx, vagina, vulva, penis, and anus. ${ }^{1}$ Men accounted for $43 \%$ of HPV-related cancer cases in the United States from 2012 to 2016, with white men having the highest rates. ${ }^{1}$ The most common type of cancer for men was oropharynx $(81 \%){ }^{1}$

The HPV vaccine is highly effective at preventing these cancers. The first HPV vaccine was licensed in

This article was externally peer reviewed.

Submitted 22 December 2019; revised 2 March 2020; accepted 4 March 2020.

From the School of Social Work, University of North Carolina at Charlotte (YG); Department of Public Health Sciences, University of North Carolina at Charlotte (JB).

Funding: None.

Conflict of interest: None.

Corresponding author: Yuqi Guo, PhD, College of Health and Human Services, 9201 University City Blvd, Room 495, Charlotte, NC 28223. (E-mail: yguo16@uncc.edu).
2006 in the United States (US). ${ }^{2}$ There are 3 currently licensed vaccines to protect against HPV infections, given in a 2-dose or 3-dose schedule, though only Gardasil-9 is distributed in the United States. ${ }^{3,4}$ These vaccines are $80 \%$ to $95 \%$ effective in preventing HPV-related cancers and notably, vaccines prevent transmission of high-risk HPV strains to sexual partners. ${ }^{4}$ However, the HPV vaccination rate is much lower in males than females. Among male adolescents 13 to 17 years, $66.3 \%$ had initiated the vaccine $\mathrm{e}^{5}$ and among men ages 18 to 32 years old, the $\mathrm{HPV}$ vaccination rate was $10.7 \%$, with a $48 \%$ vaccination completion rate. ${ }^{6}$

The Center for Disease Control and Prevention (CDC) and Advisory Committee on Immunization Practices recommend males ages 11 or 12 years old to be vaccinated. ${ }^{7}$ Males ages 13 to 26 years old should be vaccinated if not previously, particularly gay, bisexual, and other men who have sex with men, those who are transgender, and immunocompromised persons. ${ }^{7}$ In 
October 2018, the US Food and Drug Administration (FDA) expanded the approved age group for Gardasil9 among males to ages 9 to 45 years. ${ }^{8}$ Likewise, in 2019, the CDC began suggesting that clinical providers share decision making regarding HPV vaccination with males ages 26 to 45 years old. ${ }^{7}$

Much of the literature about HPV vaccination has focused on young boys or adolescents. However, there is little knowledge available regarding HPV vaccine use in the adult male population. The objectives of this study were to 1 ) estimate HPV vaccine initiation and completion rates among adult males in the United States and 2) to analyze factors associated with HPV vaccine initiation and completion among adult males. To achieve study objectives, this study conducted an analysis using data from the 2018 Behavioral Risk factor Surveillance System (BRFSS) of adult males 18 to 33 years old who were 11 to 26 years old in 2011.

\section{Literature Review}

The Systems Model of Clinical Preventive Care (SMCPC) was used as the theoretical model to analyze factors associated with HPV vaccination. ${ }^{9}$ The SMCPC has been previously used to analyze preventive health care use. ${ }^{10,11}$ The SMCPC conceptualizes factors related to clinical preventive care into 4 categories: predisposing, enabling, reinforcing, and situational factors. Predisposing factors refer to patients' health beliefs and attitudes, and demographics. Enabling factors refer to the resources that can assist patients in accessing clinical preventive care, such as income and insurance. Reinforcing factors strengthen individuals' likelihood of initiating the HPV vaccine, such as having a primary health care provider and health care utilization. Situational factors are related to correlated behaviors or health conditions that affect initiation.

\section{Predisposing Factors}

The HPV vaccine initiation is negatively associated with age among males. One study of males found lower rates of HPV vaccine initiation among those 19 to 21 and 22 to 26 years compared with males ages 14 to 18 years. ${ }^{12}$ In addition, men's HPV vaccine use was also influenced by their education. Men with a college degree were more likely to accept the HPV vaccine compared with men with a high school degree or in community college ( $74 \%$ to $78 \%$ and $33 \%$, respectively) among males aged 18 to 45 years old. ${ }^{13}$ Racial disparities in HPV vaccine use have been documented. One study suggested that racial and ethnic minority individuals are more likely to initiate the HPV vaccine but less likely to complete it in general populations. ${ }^{14}$ Hispanic men were more willing to initiate the vaccine, but black men were less willing to initiate the vaccine compared with white men. ${ }^{12,15}$ Similarly, sexual identity influences acceptability. Gay and bisexual men are more likely than heterosexual men to accept the HPV vaccine. ${ }^{16}$

\section{Enabling Factors}

Financial concerns, cost of the vaccine, and income have a small effect on HPV vaccine use. ${ }^{17,18}$ Males with public health insurance, compared with those with private health insurance, were more likely to have the HPV vaccine. ${ }^{12}$ Males in a household with an income greater than $\$ 50,000$ were less likely to initiate the vaccine. ${ }^{19}$ Logistic barriers such as hassle, time, and transportation are also negatively correlated with HPV vaccine completion among males. ${ }^{18}$

\section{Reinforcing Factors}

At the state level, having an HPV mandate increases vaccination rates. ${ }^{20}$ Among male adolescents, having 1 to 3 physician contacts in the last year was associated with greater odds of HPV vaccine initiation and completion. ${ }^{21}$ Having a primary health care provider can facilitate adolescents' HPV vaccine acceptability. ${ }^{18}$ No information on reinforcing factors focusing on adult males is available in current literature. However, having access to health care providers has been identified as an important predictor of HPV vaccination among adolescents. A lack of providers' recommendation for the HPV vaccine was more likely to be reported by unvaccinated boys' parents than unvaccinated girls' parents. ${ }^{19}$

\section{Situational Factors}

Men adults who used tobacco or were not sexually active or did not have oral sex were more likely to accept the HPV vaccine. ${ }^{15}$ A meta-analysis found that the effect size of tobacco use on HPV vaccine use was small. ${ }^{18}$ However, having more sexual partners was a significant predictor of completing/initiating HPV vaccines among males. ${ }^{15,22}$

To examine the rates of HPV vaccine initiation and completion among adult males, we analyzed a nationally representative survey. According to the SMCPC, the hypotheses of this analysis were as follows: among adult males, the likelihood of initiating the HPV vaccine would be associated negatively 
with age, African American ethnicity, Hispanic ethnicity, Asian ethnicity, people living with partner(s), high-HPV-risk behaviors, and health care cost, but the HPV vaccine likelihood would be associated positively with education levels, family income, health insurance coverage, health care satisfaction, health care utilization, and general health conditions. We also hypothesized that these factors would be associated with the HPV vaccine completion in the same directions.

\section{Methods}

\section{Data Resource}

Data for this study were taken from the 2018 Behavioral Risk factor Surveillance System (BRFSS). The BRFSS was designed to collect data on health behaviors and use of preventive services from the noninstitutionalized adult population ( $\geq 18$ years) across all states within the United States. ${ }^{23}$ By using a disproportionate stratified sample design for their landline samples, the BRFSS is the largest continuously conducted health survey system in the US, which provides nationally representative data. Based on the 2011 HPV vaccine recommendation, we focused on the upper range of the permissible male vaccination age of 26 years. During data collection in 2018, individuals aged 26 years in 2011 would have been 33 years old. Our sample included 3228 males 18 to 33 years old who participated in the 2018 BRFSS and responded to the HPV-related questions.

\section{Measures}

\section{Outcome Variables}

There are 2 outcome variables in this analysis. The first dependent variable for this study is a dichotomous variable of HPV vaccine initiation (yes/no), which describes whether respondents initiated the HPV vaccine. The BRFSS asked male participants, "Have you ever had the HPV vaccination (Gardasil)?" The second dependent variable includes response options for individuals who completed either a 2-dose or 3-dose schedule (yes/no), depending on if they initiated the vaccine sequence before or after their 15th birthday, according to the CDC's recommendation guidelines."

\section{Explanatory Variables}

The independent variables were selected based on the SMCPC. Four independent variable groups describing predisposing factors, enabling resources, reinforcing factors, and situational factors were analyzed for both dependent variables.

In this analysis, the predisposing variable group included the following demographic factors (age and race) and social-structural factors (levels of education and living with a partner $[1=$ yes and $0=$ no]). The variable "living with a partner(s)" describes people who were married or living with their partners. Dummy variables, non-Hispanic White, Black, Hispanic, and Others described self-identified ethnicity, and non-Hispanic Whites was the reference. Respondents' education level was rated from 1 to 6 : $1=$ never attended school, $2=$ elementary, $3=$ some high school, 4 = high school graduate, $5=$ some college, 6 = college graduate or higher.

The enabling variable group included family income, health care insurance, health care cost, and satisfied with the care received. Health care insurance is a dichotomous variable measuring whether in the past 12 months, the respondents ever needed to see a doctor but could not due to lacking health care insurance $(1=$ no and $0=y e s)$. Health care cost is a dichotomous variable measured whether the respondents had a time in the past 12 months when they needed to see a doctor but could not because of cost ( $1=$ affordable and $0=$ unaffordable $)$. Respondents' family income was measured from 1 to 8: $1=$ Less than $\$ 10,000,2=\$ 10,000$ to less than $\$ 15,000,3=\$ 15,000$ to less than $\$ 20,000,4=$ $\$ 20,000$ to less than $\$ 25,000,5=\$ 25,000$ to less than $\$ 35,000,6=\$ 35,000$ to less than $\$ 50,000,7=$ $\$ 50,000$ to less than $\$ 75,000,8=\$ 75,000$ or more. Satisfied with care received was a variable that measured the levels of satisfaction of received health care: $1=$ not at all satisfied, $2=$ somewhat satisfied, and 3 = very satisfied.

The reinforcing variable group included routine checkups and having a primary health care professional(s) $(1=$ yes and $0=$ no). The routine checkups measured the length of time since last routine checkup $(1=$ never, $2=5$ or more years ago, $3=$ more than 2 years but less than 5 years ago, $4=$ more than 1 year but less than 2 years ago, $5=$ anytime less than 12 months ago).

The situational variable group included highHPV-risk behaviors $(1=$ yes and $2=$ no) and general health condition. The high-HPV-risk behaviors measured whether the respondents have used intravenous drugs/substances, or have been treated for sexually transmitted diseases, or have given or received money or drugs in exchange for sex during 
the past 12 months preceding the 2018 BRFSS interview. Respondents' general health condition was measured by rating 1 to 5 ( 1 = poor, 2 = fair, $3=$ good, $4=$ very good, $5=$ excellent).

\section{Data Analysis}

General characteristics of respondents were described by calculating the frequencies, percentages, averages, and standard deviations. The associations between the independent and dependent variables were examined by 2 binomial logistic regression models. The multicollinearity diagnostic of participants was examined. No multicollinearity problem was identified in the 2 binomial logistic regression models. All analyses employed sampling weights provided by the 2018 BRFSS. Data analysis was conducted with STATA 12.0 software (StataCorp, College Station TX).

\section{Results}

\section{Descriptive Statistics}

The average age of participants was 25.86 years $(\mathrm{SD}=4.64)$ (Table 1). Of the 3228 participants,

Table 1. Sample Characteristics $(n=3228)$

\begin{tabular}{ll}
\hline Predisposing Factors & \\
\hline Age (mean, SD) & $25.86,4.64$ \\
Race (n, \%) & $1683,52.1 \%$ \\
$\quad$ non-Hispanic white & $413,12.8 \%$ \\
African American & $537,16.6 \%$ \\
Hispanic & $374,11.6 \%$ \\
Asian & $829,25.7 \%$ \\
Living with partner(s) (yes; n, \%) & $4.78,0.95$ \\
Education (mean SD) & $5.99,1.79$ \\
Enabling factors & \\
Family income (mean, SD) & $44,1.4 \%$ \\
Unable to see doctors because of & $67,2.1 \%$ \\
Health care cost (yes; $\mathrm{n}, \%)$ & $2.56,0.27$ \\
Health care insurance (yes; $\mathrm{n}, \%)$ & \\
Satisfied with care received (mean, SD) & $4.24,1.10$ \\
Reinforcing factors & $1778,55.1 \%$ \\
Routine Checkups (mean, SD) & \\
Have health care professional(s) (yes; n, \%) & \\
Situational factors & $498,15.4 \%$ \\
High-HPV-risk behaviors (yes; n, \%) & $3.70,0.96$ \\
General health condition (mean, SD) & \\
\hline
\end{tabular}

Education: 4.85 = high school graduate; family income: $6.17=$ $\$ 35,000$, to less than $\$ 50,000$; satisfied with care received: 2.56 = very satisfied; routine checkups: $4.25=$ more than 1 year but less than 2 years; general health condition: 3.62 = good. HPV, Human Papillomavirus; SD, standard deviation.
Table 2. HPV Vaccination Initiation and Completion Rates among Adult Males $(\mathrm{n}=3228)$

n, \%/Mean, SD

\begin{tabular}{lc}
\hline HPV vaccination initiation & $454,14.1 \%$ \\
rate & HPV vaccination completion \\
rate & $\begin{array}{c}161,35.5 \% \text { (of participants who } \\
\text { initiated) }\end{array}$ \\
\hline
\end{tabular}

HPV, Human Papillomavirus; SD, standard deviation.

more than half were non-Hispanic whites (52.1\%), $12.8 \%$ were African American, 16.6\% were Hispanic, and $11.6 \%$ were Asian. A quarter of participants were living with partner(s). As well, 55.1\% had health care professionals and $15.4 \%$ reported having high-HPV-risk behaviors.

Participants' average level of education was high school graduate $(\mathrm{SD}=0.95)$. Their average general health condition was good $(\mathrm{SD}=1.00)$. The average family income was $\$ 35,000$ to less than $\$ 50,000$ $(\mathrm{SD}=1.79)$. The average time of having routine checkups was more than 1 year but less than 2 years $(\mathrm{SD}=1.10)$. On average, participants were very satisfied with their health care experiences $(\mathrm{SD}=$ 0.27). Few participants reported that lack of health insurance $(\mathrm{n}=44,1.4 \%)$ or unaffordable health care costs $(n=67,2.1 \%)$ were barriers to accessing health care.

\section{HPV Vaccination Initiation and Completion Rates}

In the United States, the HPV vaccine initiation rate among males 18 to 33 years old was $14.1 \%$ (Table 2). The HPV vaccine completion rate among males ages 18 to 33 years old was $35.4 \%$ of adult males who initiated.

\section{Multivariate Analysis Results}

Among adult males in the United States, the likelihood of initiating HPV vaccine negatively associated with age (odds ratio $[\mathrm{OR}]=0.87, P<.00$ ) and living with partner(s) (odds ratio $[\mathrm{OR}]=0.53$, $P<.01$ ), but this likelihood was positively associated with the levels of education $(\mathrm{OR}=1.49, P<$ $.00)$, having routine checkups $(\mathrm{OR}=1.29, P<.05)$, having a primary health care provider $(\mathrm{OR}=1.51$, $P<.05)$, and having high-HPV-risk behaviors $(\mathrm{OR}=1.53, P<.01)$ (Table 3$)$. Among adult males in the United States, the likelihood of completing a 3 -dose HPV vaccine schedule negatively associated with age $(\mathrm{OR}=0.85, P<.00)$ but positively associated with the levels of education $(\mathrm{OR}=1.64, P<$ 
$.01)$, having routine checkups $(\mathrm{OR}=1.45, P<.05)$, and having high-HPV-risk behaviors $(\mathrm{OR}=1.79$, $P<.05)$.

\section{Discussion}

Using national vaccination data from the 2018 BRFSS, this study estimated that the HPV vaccine initiation rate among US adult males (18 to 33 years old) was $14.1 \%$ and the HPV vaccine completion rated was $35.4 \%$. This study's estimated HPV vaccination rate among adult males is higher, but the completion rate among adult males is lower than statistics reported in 2013. ${ }^{6}$ Based on these rates, we estimate that about 22.7 million adult males (18 to 33 years old) initiated HPV vaccines and 8.0 million adult males (18 to 33 years old) completed all shots of the HPV vaccine in the United States Given that the HPV vaccine can reduce the risk of genital warts, penile cancer, anal cancer, and oral cancer among adult males, and prevent the spread of HPV to their sexual partners, ${ }^{24,25}$ it is important to increase $\mathrm{HPV}$ vaccine completion rates among adult males.

\section{Predisposing Factor}

The results of our analyses partially supported our hypothesis. HPV vaccine initiation and completion were both negatively associated with age. According to the CDC's recommendation, age is the most important factor of receiving HPV vaccines. People are recommended to initiate HPV vaccine as early as possible after 11 years old. Therefore, older adult males were significantly less likely to have the HPV vaccine. In addition, adult males who were married were less likely to be vaccinated for HPV. Marriage can reduce the likelihood of initiating an HPV vaccine, and there are 2 plausible reasons. First, marital status is positively associated with age. Second, HPV vaccine is targeting primarily those at-risk for sexually transmitted infections, and having a stable sexual relationship with a partner may undermine vaccination. ${ }^{25}$ However, our analysis suggested that if a male had initiated the HPV vaccine, living with a partner would not reduce their likelihood of completing all shots of HPV vaccines.

Education was identified as a significant factor positively associated with HPV vaccine initiation and completion among adult males. Men with higher levels of education were more likely to initiate an HPV vaccine. Previous studies suggested that men with higher levels of education have higher acceptance of the HPV vaccine. ${ }^{15,22}$ The potential explanation may be that people with higher levels of education have more exposure to HPV knowledge and have a higher awareness of the HPV vaccine. ${ }^{22}$

Notably, we found no evidence of racial disparities in HPV vaccine initiation nor completion among males. ${ }^{26}$ African Americans and other minority groups are disproportionately affected by the $\mathrm{HPV}$ infection. ${ }^{5}$ If vaccination rates by race do not change drastically, then we may expect a decrease in the number of HPV infections among males.

\section{Reinforcing Factors}

Having routine checkups and a primary doctor are important factors for males' HPV vaccine use. Males who had routine checkups more often were more likely to complete the scheduled doses of $\mathrm{HPV}$ vaccine. A systematic review of factors associated with HPV vaccine uptake demonstrated that more health care utilization and having a health care provider could facilitate HPV vaccine uptake. ${ }^{27}$ Health care providers play an essential role in providing HPV-related information and assisting patients in initiating the vaccine series. ${ }^{27}$ Another prior study reported that the participants who received a provider recommendation for the HPV vaccine were 4 times more likely to receive an HPV vaccine than the participants who did not receive a recommendation. ${ }^{28}$ Visiting doctors for routine checkups and/or having a health care provider may provide more opportunities for receiving HPV-related knowledge and HPV vaccine information.

\section{Situational Factors}

Men with high-HPV-risk behaviors (injection drug use, having sexually transmitted disease(s), and exchanging money or goods for sex) were more likely to be vaccinated, which was a promising finding regarding the importance of vaccinating men at high risk of HPV infection. This finding suggested that public health vaccination efforts targeting populations at high risk of HPV infection have been successful. Injection drug use, having sexually transmitted disease(s), and engaging in sex work can significantly increase the risk of HPV infection. ${ }^{29}$ Therefore, the CDC pays extra attention to sexual minority men and immunocompromised men 
(including those with HIV infection) by adjusting their recommended age up to 45 years old for vaccination. ${ }^{7}$ In addition, men with high HPV risk are more likely to receive HPV vaccine recommendations from health care providers, which potentially increases the likelihood of HPV vaccine initiation. ${ }^{30}$

\section{Limitation}

The study findings should be interpreted within its limitations. First, this analysis did not focus on males regarding to the FDA's recent age updates. This study analyzed factors associated with adult males' HPV vaccination status. To better understand adult males' HPV vaccination behaviors, future studies should focus on males who received HPV vaccinates in their adulthood (who ages 18 to 45 years old). Second, this study could not analyze the role of health literacy on HPV vaccination among US men. The 2018 BRFSS did not measure HPV-related knowledge, beliefs, attitudes, and HPV vaccine awareness, even though these factors are docu- mented in the literature as strong predictors of accepting the HPV vaccine among general populations. Second, we were not able to identify the gay, bisexual, and other men who have sex with men, transgender people, and immunocompromised persons (including those with HIV infection) who were highly recommended by the CDC for HPV vaccines. Future studies may focus on these populations who are at high risk of HPV infection. Despite these limitations, our study estimated the national HPV vaccination rate among adult males by using data from the first year of BRFSS HPV vaccination data and reported adult males' HPV vaccination.

\section{Conclusions}

In this study, we reported the HPV vaccination rate among adult men in the United States and analyzed factors associated with HPV vaccine initiation and completion among males aged 18 to 33 years old. Having the HPV vaccination is not only beneficial for preventing cancer-causing infections and pre-

Table 3. Factors Associated with HPV Vaccination Initiation and Completion $(\mathbf{n}=3228)$

\begin{tabular}{|c|c|c|c|c|}
\hline & \multicolumn{2}{|c|}{ HPV Vaccine Initiation } & \multicolumn{2}{|c|}{ HPV Vaccine Completion } \\
\hline & Odds Ratio & RSE & Odds Ratio & RSE \\
\hline \multicolumn{5}{|l|}{ Predisposing Factors } \\
\hline Age & $0.87^{*}$ & 0.02 & $0.85^{*}$ & 0.02 \\
\hline \multicolumn{5}{|l|}{ Race (non-Hispanic Whites) } \\
\hline African American & 1.06 & 0.25 & 0.87 & 0.37 \\
\hline Hispanic & 0.88 & 0.27 & 0.71 & 0.42 \\
\hline Asian & 1.71 & 0.69 & 0.87 & 0.39 \\
\hline Living with partner(s) (single) & $0.52^{\dagger}$ & 0.02 & 0.85 & 0.26 \\
\hline Education & $1.49^{*}$ & 0.17 & $1.64^{\dagger}$ & 0.31 \\
\hline \multicolumn{5}{|l|}{ Enabling Factors } \\
\hline Family income & 0.94 & 0.06 & 0.97 & 0.07 \\
\hline Health care insurance (not a barrier) & 0.61 & 0.42 & 0.83 & 0.70 \\
\hline Healthcare cost (not a barrier) & 0.61 & 0.32 & 0.59 & 0.61 \\
\hline Satisfied with care received & 1.25 & 0.32 & 1.50 & 0.50 \\
\hline \multicolumn{5}{|l|}{ Reinforcing Factors } \\
\hline Routine Checkups & $1.29^{\ddagger}$ & 0.15 & $1.45^{\ddagger}$ & 0.25 \\
\hline Have health care professional(s) (no) & $1.51^{\ddagger}$ & 0.33 & 1.49 & 0.51 \\
\hline \multicolumn{5}{|l|}{ Situational Factors } \\
\hline High-HPV-risk behaviors (no) & $1.53^{\ddagger}$ & 0.35 & $1.79^{\ddagger}$ & 0.58 \\
\hline General health condition & 1.04 & 0.11 & 1.06 & 0.14 \\
\hline Wald's $\chi^{2}=105.51, P=.00$, Wald's $\chi^{2}=$ & & & & \\
\hline
\end{tabular}

RSE, robust standard error; HPV, Human Papillomavirus.

${ }^{*} P<.00$.

${ }^{\dagger} P<.01$.

${ }^{\ddagger} P<.05$. 
cancers in males but is also an effective strategy to stop the spreading of HPV infection to their sexual partners. In this study, we found that males of younger age, with higher education levels, who more often participate in routine checkups, who have a health care provider, and who engage in high-HPV-risk behaviors were more likely to have initiated the HPV vaccine. Furthermore, men with higher education levels, more frequent routine checkups, and who engage in high-HPV-risk behaviors were more likely to have completed the HPV vaccines. To promote the prevention of HPVrelated diseases, it is important to encourage adult males to designate a primary care provider and have routine checkups. These protective factors are significant in the promotion of HPV vaccination among males. Promoting access to health care resources may improve current HPV vaccination rates among males. Financial incentives, such as cost-reduction strategies or efforts to limit out-ofpocket costs may effectively increase the HPV vaccination rate among males in the US In addition, it is important for health care providers to share information regarding HPV vaccines with adult males who experience any of the factors related to HPV vaccination or infection.

In addition, the findings of our study confirmed that public health systems should focus on vaccinating males at high risk of HPV infection, including people with a history of injection drug use, sexually transmitted infection(s), or exchanging money or goods for sex. To maximize the HPV vaccination coverage among males, US health care systems should consider encouraging health care practitioners to recommend the HPV vaccination to males at high risk of HPV infection across various health care settings, including primary care facilities and emergency rooms. Possible areas for future research should include studying HPV vaccination among gay, bisexual, and men who have sex with men, transgender people, and people infected with HIV. Effectively increasing HPV vaccination among men in the United States requires effort in both macro (health care policy and health care systems) and micro (health care providers) practice (Table 3 ).

To see this article online, please go to: http://jabfm.org/content/ 33/4/592.full.

\section{References}

1. Centers for Disease Control and Prevention. Cancers Associated with Human Papillomavirus,
United States-2012-2016 | CDC. U.S. Cancer Statistics Data Briefs. https://www.cdc.gov/cancer/ uscs/about/data-briefs/no10-hpv-assoc-cancersUnitedStates-2012-2016.htm. Published 2019. Accessed October 17, 2019.

2. McLemore MR. Gardasil: Introducing the New Human Papillomavirus Vaccine. Clin J Oncol Nurs 2006;10:559-60.

3. Tumban E, Peabody J, Tyler M, Peabody DS, Chackerian B. VLPs displaying a single L2 epitope induce broadly cross-neutralizing antibodies against human papillomavirus. PLoS One 2012;7:e49751.

4. Zhai L, Tumban E. Gardasil-9: a global survey of projected efficacy. Antiviral Res 2016;130:101-9.

5. Walker TY, Elam-Evans LD, Yankey D, et al. National, regional, state, and selected local area vaccination coverage among adolescents aged 1317 years-United States, 2018. MMWR Morb Mortal Wkly Rep 2019;68:718-23.

6. Han JJ, Beltran TH, Song JW, Klaric J, Choi YS. Prevalence of genital human papillomavirus infection and human papillomavirus vaccination rates among US adult men. JAMA Oncol 2017;3:810.

7. Centers for Disease Control and Prevention (CDC). Recommendations on the use of quadrivalent human papillomavirus vaccine in males-Advisory Committee on Immunization Practices (ACIP), 2011. Morbid Mortal Week Rep 2011;60:1705.

8. US Food and Drug Administration. FDA approves expanded use of Gardasil 9 to include individuals 27 through 45 years old. 2018. https://www.fda.gov/ news-events/press-announcements/fda-approvesexpanded-use-gardasil-9-include-individuals-27through-45-years-old. Accessed October 17, 2019.

9. Walsh JM, McPhee SJ. A systems model of clinical preventive care: an analysis of factors influencing patient and physician. Health Educ Q 1992;19:157-75.

10. Guo Y, Sims OT. HCV health policy developments in response to the national viral hepatitis action plan: a brief update. Soc Work Public Health 2017;32:77-81.

11. Guo Y, Cheng TC, Yun Lee H. Factors associated with adherence to preventive breast cancer screenings among middle-aged African American women. Soc Work Public Health 2019;34:646-56.

12. Thomas R, Higgins L, Widdice L, Chandler E, Ding L, Kahn J. Factors associated with HPV vaccination and accuracy of HPV vaccination self-report among 13 to 26 year-old men. J Adolesc Health 2016;12:819-27.

13. Liddon N, Hood J, Wynn BA, Markowitz LE. Acceptability of human papillomavirus vaccine for males: a review of the literature. J Adolesc Health 2010;46:113-23.

14. Spencer JC, Calo WA, Brewer NT. Disparities and reverse disparities in HPV vaccination: a systematic review and meta-analysis. Prev Med (Baltim) 2019;123:197-203. 
15. Ferris DG, Waller JL, Miller J, et al. Variables associated with human papillomavirus (HPV) vaccine acceptance by men. J Am Board Fam Med 2009;22:34-42.

16. Gilbert P, Brewer NT, Reiter PL, Ng TW, Smith JS. HPV vaccine acceptability in heterosexual, gay, and bisexual men. Am J Mens Health 2011;5:297305.

17. Dibble KE, Maksut JL, Siembida EJ, Hutchison M, Bellizzi KM. A systematic literature review of HPV vaccination barriers among adolescent and young adult males. J Adolesc Young Adult Oncol 2019;8:495-511.

18. Newman PA, Logie CH, Doukas N, Asakura K. HPV vaccine acceptability among men: a systematic review and meta-analysis. Sex Transm Infect 2013;89:568-74.

19. Gilkey MB, Moss JL, McRee AL, Brewer NT. Do correlates of HPV vaccine initiation differ between adolescent boys and girls? Vaccine 2012; 30:5928-34.

20. Franco M, Mazzucca S, Padek M, Brownson RC. Going beyond the individual: how state-level characteristics relate to HPV vaccine rates in the United States. BMC Public Health 2019;19:246.

21. Lu PJ, Yankey D, Jeyarajah J, et al. HPV vaccination coverage of male adolescents in the United States. Pediatrics 2015;136:839-49.

22. Jones M, Cook R. Intent to receive an HPV vaccine among university men and women and implications for vaccine administration. J Am Coll Health 2008;57:23-31. doi:10.3200/JACH.57.1.23-32

23. US Centers for Disease Control and Prevention. The Behavioral Risk Factor Surveillance System
(BRFSS) OVERVIEW: BRFSS 2018.; 2019. https://www.cdc.gov/brfss/annual_data/2018/pdf/ overview-2018-508.pdf.

24. Petrosky E, Bocchini JA, Hariri S, et al. Use of 9valent human papillomavirus (HPV) vaccine: updated HPV vaccination recommendations of the advisory committee on immunization practices. Morb Mortal Wkly Rep 2015;64:300-4.

25. Bosch FX, Lorincz A, Muñoz N, Meijer C, Shah $\mathrm{KV}$. The causal relation between human papillomavirus and cervical cancer. J Clin Pathol 2002;55: 244-65.

26. Brewer NT, Fazekas KI. Predictors of HPV vaccine acceptability: a theory-informed, systematic review. Prev Med 2007;45:107-14.

27. Kessels SJM, Marshall HS, Watson M, BraunackMayer AJ, Reuzel R, Tooher RL. Factors associated with HPV vaccine uptake in teenage girls: a systematic review. Vaccine 2012;30:3546-56.

28. Ylitalo KR, Lee H, Mehta NK. Health care provider recommendation, human papillomavirus vaccination, and race/ethnicity in the US national immunization survey. Am J Public Health 2013;103: 164-9.

29. Smith-McCune KK, Shiboski S, Chirenje MZ, et al. Type-specific cervico-vaginal human papillomavirus infection increases risk of HIV acquisition independent of other sexually transmitted infections. PLoS One 2010;5:e10094.

30. Meites E, Szilagyi PG, Chesson HW, Unger ER, Romero JR, Markowitz LE. Human papillomavirus vaccination for adults: updated recommendations of the Advisory Committee on Immunization Practices. Am J Transplant 2019;68:698-702. 